\title{
The Highlights of Cardiovascular Disease Depend on Epidemiological Statistics
}

\section{Abstract}

Epidemiology contributes to assessing and clarify the role and significance of the cardiovascular risk factor analysis of statistical methodologies. Primarily, ischemic and hypertensive heart disease is the pathological conditions more widely examined were the major outcomes able to determine an unfavorable prognosis for those individuals who are suffering [1-3].

Therefore, this editorial focuses the highlights emerged in the current studies.

Until a few years ago, mathematical models were applied to the statistical procedures. These methods used empirical equations that determined how a system changed from a result to other similar and/or of what one variable could influence the estimate of observations instead of other variables. By these procedures can be classified a largest number of subjects with the same, or almost same, disease.

Statistical models provided the characterization of numerical results by estimating probabilistic values that, therefore, have needed continuously to be updated. This is a consequence of the fact that the number of observations conducted involved only a limited aspect of the findings linked with what the risk factor influenced the cardiovascular disease and, in addition, always newer reports have been published.

For an example, a risk factor like smoking could be approached in different types of study -clinical, biological, metabolic and many others, which were independent one to another- that, however, gave partial results of the pathological observations identified. Notwithstanding these observations, partially limited when compared to those documented today, the American Heart Association included both active and passive smoking among the major risk factors for heart disease in both adults and children [4]. There was evidence that the wide number of studies conducted, even if examined separately, permitted to identify the mechanisms of cardiovascular damage caused by cigarette smoking using mathematical calculation providing often similar results derived by different approaches to the study protocol.

Today, the progress observed by statistical approaches permits a more appropriate knowledge of the role and significance of the risk factors related to the cardiovascular disease. Randomized controlled clinical and pathological studies in cardiovascular patients have undoubtedly demonstrated conflicting, but also similar, the results of the characteristics and progression of heart and blood vessel disease.

Primarily, statistical analysis demonstrated that a multifactorial intervention characterizes the role of cardiovascular risk factors in determining a heart disease. Moreover, studies documented cigarette

\section{Conclusion}

In conclusion, there is evidence that the epidemiological observations are the basis to learn the highlights that cardiovascular findings continuously provide.
Editorial

Volume 10 Issue 3 - 2017

Aurelio Leone MD $^{1,2,3 *}$

${ }^{1}$ Fellow of the American Society of Hypertension (FASH), USA ${ }^{2}$ Fellow of the Royal Society for Promotion of Health (FRSPH), $U K$

${ }^{3}$ Editor-in-Chief of the Journal of Cardiology \& Current Research, USA

*Corresponding author: Aurelio Leone, Fellow of the American Society of Hypertension (USA), Fellow of the Royal Society for Promotion of Health (UK), Editor-in-Chief of the Journal of Cardiology \& Current Research (USA), Via Provinciale 27, 19030 Castelnuovo Magra, Italy,

Email: reliol@libero.it

Received: December 18, 2017 | Published: December 29, 2017

smoking as an etiologic factor for cardiovascular disease, including ischemic and hypertensive heart disease [5,6].

Generally, cardiovascular risk factors, all together, show contributing the formation and evolution of some typically functional and morphological alterations of the cardiovascular system, primarily atherosclerotic plaque, which is the most important pathology of the arterial vessels $[7,8]$.

From these observations, there is no doubt that the epidemiological survey has achieved a new positive impulse from the analysis provided by these electronic devices. It is worth noting that WHO [9] defines the epidemiology as the study of the distribution and determinants of healthrelated states or events (including heart disease), and the application of this study to the control of diseases and other health problems. Various methods can be used to carry out epidemiological investigations: surveillance and descriptive studies can be used to assess the distribution of the data, while analytical studies are used to study the determinants. Therefore, there is evidence that having analyzed and stored observations related to a specific topic, including the results derived from the relationship between cardiovascular risk factors and heart disease, is a basic parameter to assess correctly the existing links. In addition, metaanalysis studies [10] have widened the spectrum of the cardiovascular interventions on patients suffering from cardiovascular disease.

\section{References}

1. Leone A (2003) Relationship between cigarette smoking and other coronary risk factors in atherosclerosis: risk of cardiovascular disease and preventive measures. Curr Pharm Des 9 (29): 2417-2423. 
2. Elwood P (2001) An update on risk factors for vascular disease. Pharm J 267: 194-196.

3. Leone A (2016) Markers of atherosclerotic disease: What do they mean? Current opinion and future trends. Curr Pharm Des 22(1) 7-17.

4. Glantz SA, Parmley WW (1985) Passive smoking and heart disease. JAMA 273(13): 1047-1053.

5. Leone A (2015) Should smoke exposure be considered an etiologic factor for cardiovascular disease? Future Sci. OA 10.4155/ fsoa.2015.0018.

6. Leone A, Landini L, Leone A (2010) What is tobacco smoke? Sociocultural dimensions of the association with cardiovascular risk Curr Pharm Des 16(23): 2510-2517.
7. Ross R (1999) Atherosclerosis- An inflammatory disease. N Engl J Med 340(2): 115-126.

8. Libby P, Ridker PM, Maseri A (2002) Inflammation and atherosclerosis. Circulation 105: 1135-1143.

9. WHO (2010) Health and Epidemiology. WHO.

10. Leone A (2012) Statistics for understanding damage. In: Passive Smoking and Cardiovascular Pathology. Mechanisms and Physiopathological Bases of Damage, A Leone Editor, Cardiology Research and Clinical Developments, Nova Biomedical New York, USA, pp. 141-153. 